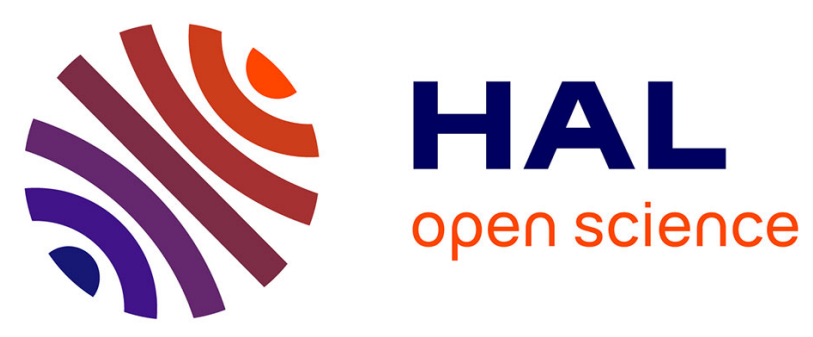

\title{
Improving vision for surgeons during laparoscopy: the Enhanced Laparoscopic Vision System ELViS
}

Bertrand Trilling, Adrian Mancini, Gaëlle Fiard, Pierre Alain Barraud,

Marion Decrouez, Sinara Vijayan, Mathias Tummers, Jean Luc Faucheron, Sophie Silvent, Christel Schwartz, et al.

\section{To cite this version:}

Bertrand Trilling, Adrian Mancini, Gaëlle Fiard, Pierre Alain Barraud, Marion Decrouez, et al.. Improving vision for surgeons during laparoscopy: the Enhanced Laparoscopic Vision System ELViS. Surgical Endoscopy, 2021, 35 (5), pp.2403-2415. 10.1007/s00464-021-08369-2 . inserm-03208307

\section{HAL Id: inserm-03208307 https://www.hal.inserm.fr/inserm-03208307}

Submitted on 26 Apr 2021

HAL is a multi-disciplinary open access archive for the deposit and dissemination of scientific research documents, whether they are published or not. The documents may come from teaching and research institutions in France or abroad, or from public or private research centers.
L'archive ouverte pluridisciplinaire HAL, est destinée au dépôt et à la diffusion de documents scientifiques de niveau recherche, publiés ou non, émanant des établissements d'enseignement et de recherche français ou étrangers, des laboratoires publics ou privés. 


\title{
Improving vision for surgeons during laparoscopy: the Enhanced Laparoscopic Vision System ELViS
}

\author{
ELViS: visually-enhanced laparoscopy \\ Bertrand TRILLING MD PhD ${ }^{1,2}$, Adrian MANCINI MD ${ }^{1,2}$, Gaëlle FIARD MD PhD ${ }^{2,3}$, Pierre Alain \\ BARRAUD ${ }^{1}$, Marion DECROUEZ PhD ${ }^{4}$, Sinara VIJAYAN PhD ${ }^{1}$, Mathias TUMMERS ${ }^{1}$, Jean Luc \\ FAUCHERON MD PhD ${ }^{1,2}$, Sophie SILVENT PhD 5 , Christel SCHWARTZ ${ }^{5}$, Sandrine Voros PhD ${ }^{1,6}$
}

1 Grenoble Alps University, National Centre for Scientific Research, Grenoble National Polytechnical Institute, Grenoble Alps University Hospital, TIMC-IMAG Laboratory, Grenoble, France

2 Colorectal Surgery Unit, Visceral surgery and Acute care surgery Department, Grenoble Alps University Hospital, Grenoble, France

3 Urology, Grenoble Alps University Hospital, Grenoble, France

4 Surgiqual Institute, Meylan, France

5 Investigation Clinical Center 1406, University Grenoble Alpes, Grenoble, France ; Investigation Clinical Center 1406, National Institute of Health and Medical Research, Grenoble, France;

Technological Innovation - Investigation Clinical Center, Department of Research, University Hospital, Grenoble, France

6 National Institute of Health and Medical Research

Author to whom correspondence should be addressed:

Dr Bertrand TRILLING MD PhD

Service de Chirurgie Viscérale et de l'Urgence

CHU Grenoble Alpes

Boulevard de la Chantourne

38000 Grenoble Cedex

Btrilling@chu-grenoble.fr

Tel : +33476768884

Funding information: The work described in this paper was funded by the French National Agency for Research (Agence Nationale de la Recherche) as part of the DEPORRA2 project (reference ANR-14CE17-0009) and by the French government's Investissements d'Avenir programme as part of the Labex CAMI (reference ANR-11-LABX-0004) 


\section{Abstract}

Background: For many abdominal surgical interventions, laparotomy has gradually been replaced by laparoscopy, with numerous benefits for the patient in terms of post-operative recovery. However, during laparoscopy, the endoscope only provides a single viewpoint to the surgeon, leaving numerous blind-spots and opening the way to peri-operative adverse events. Alternative camera systems have been proposed, but many lack the requisite resolution/robustness for use during surgery or cannot provide real-time images. Here, we present the added value of the Enhanced Laparoscopic Vision System (ELViS) which overcomes these limitations and provides a broad view of the surgical field in addition to the usual high-resolution endoscope.

Methods: Experienced laparoscopy surgeons performed several typical procedure steps on a live pig model. The time-to-completion for surgical exercises performed by conventional endoscopy and ELViSassisted surgery was measured. A debriefing interview following each operating session was conducted by an ergonomist, and a System Usability Scale (SUS) score was determined.

Results: Proof of concept of ELVIS was achieved in an animal model with 7 expert surgeons without peroperative adverse events related to the surgical device. No differences were found in time-tocompletion. Mean SUS score was 74.7, classifying the usability of the ELViS as "good". During the debriefing interview, surgeons highlighted several situations where the ELViS provided a real advantage (such as during instrument insertion, exploration of the abdominal cavity or for orientation during close work), and also suggested avenues for improvement of the system.

Conclusions: This first test of the ELViS prototype on a live animal model demonstrated its usability and provided promising and useful feedback for further development.

Keywords : Distributed Laparoscopy, Trocar prototype, Animal model, Phase 0 medical device evaluation, Enhanced visualisation 


\section{Introduction}

In abdominal surgery, laparotomy was long considered the optimal approach. It allows direct access to the visceral organs through a large incision in the abdominal wall, and analysis of the surgical area by palpation, while providing the surgeon with a full view of the whole abdominal cavity throughout the procedure. The development of minimally invasive surgeries such as laparoscopic surgery was a real revolution in the surgical management of numerous disorders. Through this approach, the surgical procedure is performed using instruments introduced into the abdominal cavity through trocars with internal diameters of between $5 \mathrm{~mm}$ and $15 \mathrm{~mm}$. After insufflating the abdominal cavity with carbon dioxide, an endoscope (small-diameter extended lens) connected to a camera is inserted through the optical trocar, often located beside the umbilicus, and held by the surgical assistant.

Compared to laparotomy, laparoscopic surgery has several advantages [1]. As the incisions are smaller, parietal trauma is reduced, particularly for obese patients, and intra-abdominal contamination is limited. The surgery tends to be less haemorrhagic [2] since the dissection can be more precise thanks to the magnification of the surgical area by the endoscope. Post-operative intra-abdominal adhesions are less frequent [3]. Post-operative return to activity is faster [4]. Oncological results are similar [2,5]. And finally, the aesthetic advantages are considerable, once again thanks to the much smaller size of incisions.

Despite these advantages, laparoscopy presents disadvantages in terms of perioperative safety during the intervention and ease-of-work for the surgeon. For example, during its initial development, the rate of injuries to the biliary tract during laparoscopic cholecystectomy increased considerably compared to open surgery $[6,7]$. This increase could be linked to the learning curve of the technique, but also to the constraints specific to laparoscopy, which include loss of hand-eye coordination or a reduced field of view. The presence of blind spots in the abdomen (absence of visibility in contrast to during laparotomy) is a known problem in laparoscopic surgery. Thus, when the endoscope is zoomed to magnify the image of the surgical area, the surrounding organs are no longer visible on the image. This situation can be a source of risk and anxiety for the surgeon. In addition, as the main endoscope is usually focused and zoomed on the area in which the surgeon is working, the extremities of instruments are not visible as they pass through the abdominal region during insertion before they reach the surgical area. Consequently, organs could be injured as a result of uncontrolled movements during instrument insertion, for example causing injury to the digestive tract $[8,9]$, which may go 
unnoticed. Intraoperative adverse events appear relatively frequent and are little documented, and near misses are associated with worse patient outcomes [10,11].

During laparoscopy, another difficulty is encountered by the assistant who must present an appropriate laparoscopic image, centred on the surgical area with the correct focus and horizon lines with respect to the patient. Indeed, one of the major difficulties for novice assistants is to provide an image where the axis (horizon line) is aligned with the surgeon's axis of vision or the horizon line relative to the patient $[12,13]$. This exercise is made even more complex by the fact that magnified endoscopic images do not provide much contextual information. A lack of visuospatial concordance can create alignment defects requiring additional psychomotor efforts. The duration of surgery and the number of errors increase when the endoscope's rotational axis is not aligned with the surgical area [14].

Distributed Laparoscopy was developed in an attempt to overcome these constraints, by bringing additional cameras into the abdominal cavity. As part of this approach, the Enhanced Laparoscopic Vision System (ELViS) was developed at the TIMC (Translational Innovation in Medicine and Complexity) laboratory (Grenoble, France). ELViS allows surgeons to view the zone surrounding the surgical area from a distance. At all times, the additional images provided should allow the operator to see the "blind spots" and guide the assistant holding the camera. The User Interface and the characteristics of the ELViS were developed on a test bench to identify the optimal configuration. A preliminary study describing bench experiments was recently published[15]. The results showed that an adverse event could be detected earlier when using ELViS. Tests on a cadaver during prototype development were encouraging, but this model fails to reproduce real-life use conditions for the device (due to rigor mortis and difficulties with the pneumoperitoneum).

The present work presents a proof of concept of the ELViS on a live porcine model as a preliminary step to its transfer for use in human surgery.

\section{Materials and methods}

\section{A. ELViS prototype}

The ELViS is an improved optical trocar, developed from previously published work [16-18] from which two side cameras can be deployed (Figure 1). The shaft of the device allows the insertion of a conventional 10-mm-diameter endoscope. At its distal end, a camera module containing the two miniaturized cameras can pivot on an axis around the trocar shaft. The two mini cameras (Misumic) TD-VBL31105L-77; MISUMI Electronics Corp, Taipei City, Taiwan) are equipped with a LED illumination system to provide side images. Their specifications are summarized in Table 1. 


\begin{tabular}{ll}
\hline Size & $4.5 \times 15 \mathrm{~mm}$ \\
\hline Image resolution & $1280 \times 720$ pixels \\
\hline Horizontal visual field & $70^{\circ}$ \\
\hline Vertical visual field & $42.7^{\circ}$ \\
\hline Pace & 30 images $/ \mathrm{s}$ \\
\hline Illumination (6 LED) & 5400 Lux \\
\hline Price & $256 €$ \\
\hline
\end{tabular}

Table 1. Summary characteristics of Misumi TD-VBL31105L-77 LED-integrated cameras.

The trocar is airtight thanks to an internal valve. In the un-deployed configuration, the ELViS is inserted through a 15-mm thoracic trocar (Thoracoport ${ }^{\mathrm{TM}}$, Covidien, Minneapolis, USA) previously introduced through the abdominal wall under visual control (supplementary video). The additional $15-\mathrm{mm}$ trocar facilitates the insertion and extraction of ELViS without soiling the side cameras or injuring the abdominal wall. The 15-mm thoracic trocar is shorter than a standard 15-mm laparoscopic trocar because it does not include the insufflation system, surgeons can thus fully benefit from the overall vision offered by ELViS. After retracting the endoscope, the ELViS camera module is folded by pulling on a cable to reposition it in the axis of insertion.

The 15-mm thoracic ports are fitted with a sealing ring to provide an air-tight seal between the ELViS and the inside of the shaft. However, as thoracic trocar valves are not designed to withstand high pressure - since thoracoscopy does not require gas insufflation - we manufactured a device with dimensions similar to a thoracic trocar but fitted with an airtight diaphragm valve (MiniValve.com, Oldenzaal, The Netherlands).

The sleeve, the camera module and the deployment system are made of surgical steel, thus the mobile parts of the ELViS are resistant and hardwearing. The head of the trocar, where the silicone valves are located, is made of polyamide. These valves make it possible for the $10-\mathrm{mm}$ cameras to zoom in and out without friction or gas leak such as would occur when using a classical optical port. The structures of the ELViS are bolted and glued, and airtightness is achieved using silicone sealant.

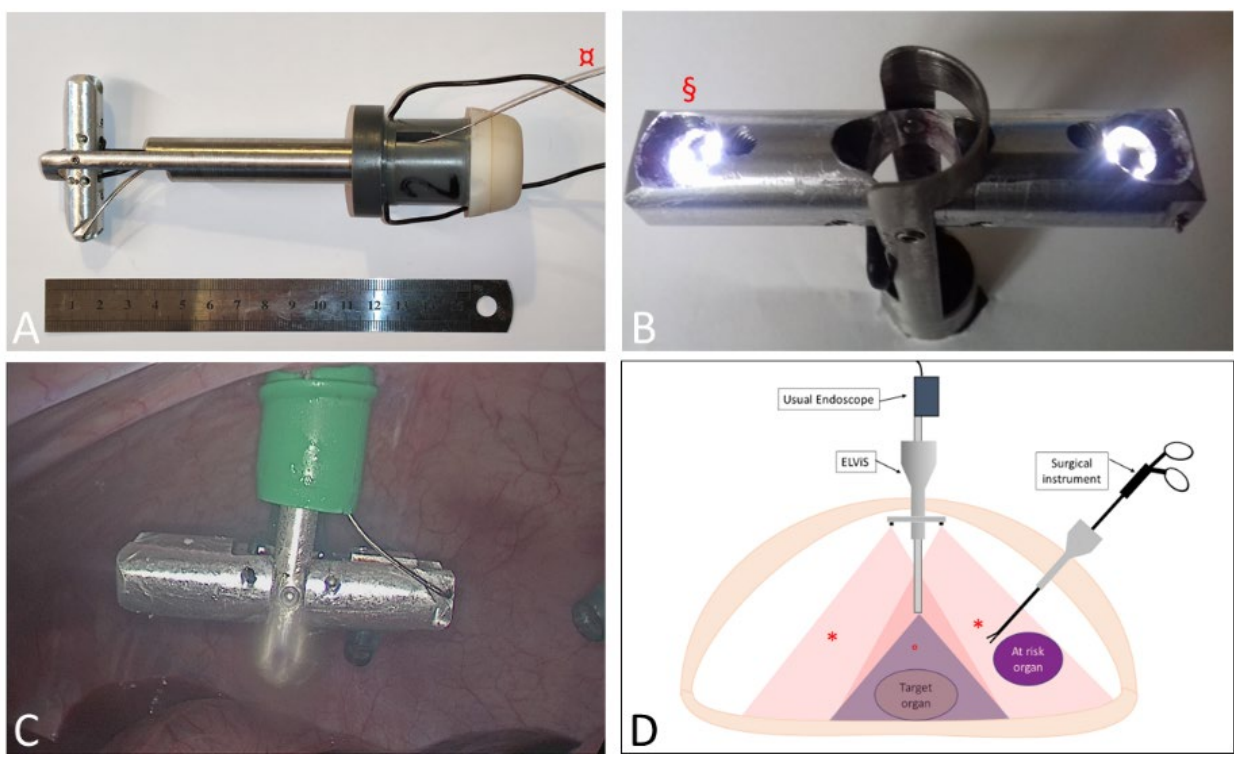


Figure 1. ELViS prototype. A. Side view of the undeployed ELViS. The ELViS is composed of three parts. The body of the trocar includes the deployment mechanism for the camera head, the camera cables and the valve system to ensure airtightness. The tubular sleeve is inserted through the $15-\mathrm{mm}$ thoracic trocar. The camera module pivots on its axis in response to traction on a rigid wire (ฉ). B. View from below of the deployed ELViS.

The lateral cameras include LED $(\S)$ to illuminate the zone surrounding the surgical area. The conventional endoscope is inserted into the trocar and passes through the camera module. C. ELViS deployed in a pig. The ELViS is inserted into the abdominal cavity through a $15-\mathrm{mm}$ trocar. D. Schematic representation of the ELViS during surgery. The endoscope is placed close to the surgical area and the surrounding zone is not included in

its visual field $\left({ }^{\circ}\right)$. The two lateral cameras on the ELViS, positioned close to the abdominal wall, extend the surgeon's field of vision $(*)$, allowing observation, when necessary, of the lateral zones representing a risk.

\section{B. Experimental model}

The laparoscopic model used in this study was the domestic pig (Sus scrofa domesticus). The equipment consisted of instruments, clamps and staplers commonly used in human surgery, and a laparoscopy column displaying the video feed of the endoscope. The experimental setup is presented in Figure 2. Each camera was connected to a central processing unit to allow display of the image on the lateral screens (21-inch computer screens), that were placed on either side of and at the same height as the screen displaying the endoscope feed. The video feeds from the three endoscopic images were stored in the hard disk of the central processing unit. The tower was mobile and could be moved during the experiment to ensure optimal ergonomic conditions for the surgical team throughout the procedure.
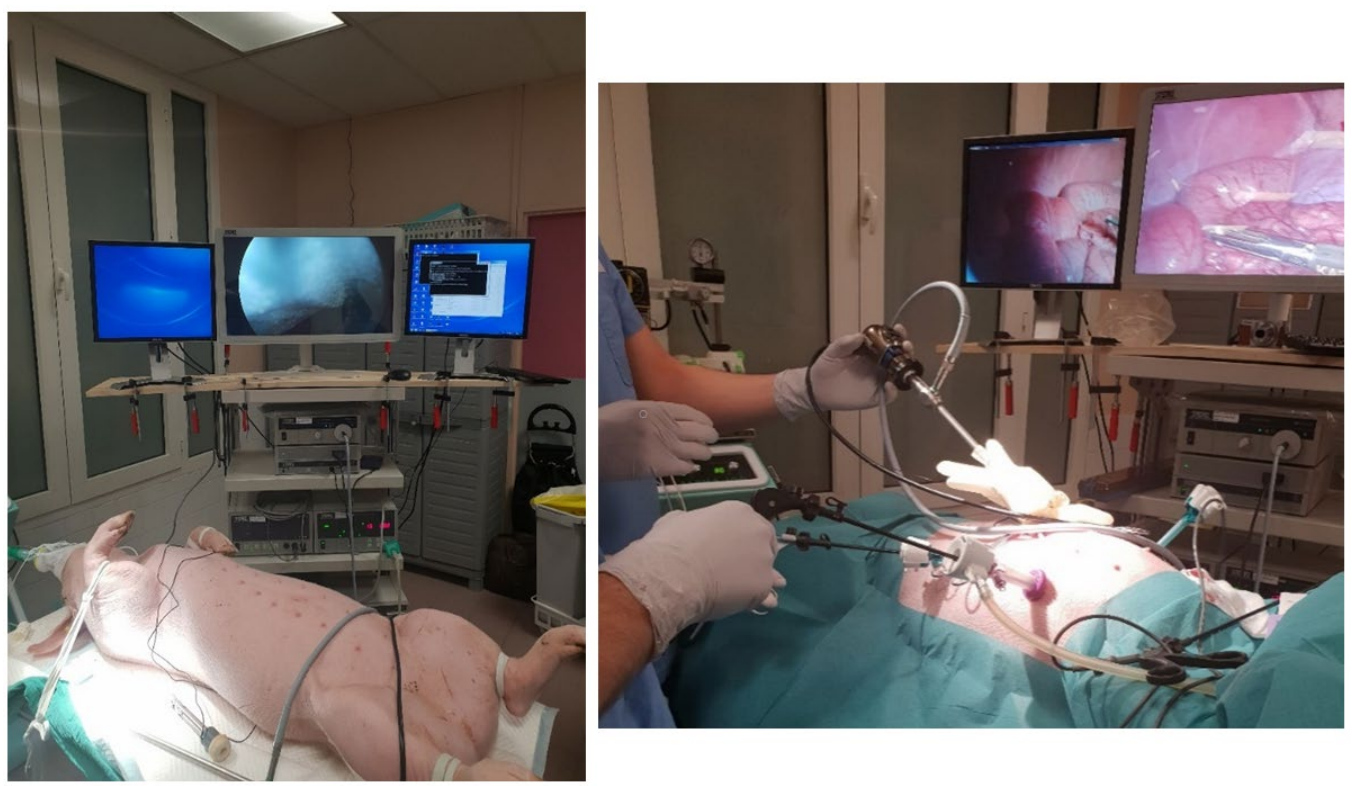

Figure 2. Installation of material for experiments on pigs. The two additional screens were placed on either side of the main screen. The laparoscopy tower included the usual material. The pig was secured with restraints after orotracheal intubation. 
Animals were anaesthetised according to the standard institutional protocol. At the end of the intervention, the animal was sacrificed by intravenous injection of a bolus of propofol administered by the veterinary team. The experimental protocol was drawn up in line with the NC3R ARRIVE recommendations [19] regulating the presentation of results from animal experiments.

The pig was placed on its back at $0^{\circ}$. The trotters were secured with restraints attached to the operating table (Figure 2). An incision was made above the umbilicus to access the peritoneal cavity. Then, a standard 10-mm umbilical trocar was inserted to explore the abdominal cavity after insufflation, and to place the additional trocars (three 5-mm trocars in the right and left hypochondrium and left flank, and one 12-mm trocar in the right flank to insert an endo-stapler or the endoscope). The insufflation tube was attached to one of the instrumental trocars. The endoscope was inserted into the 12-mm trocar, located in the right flank, to monitor the placement of the ELViS: the 15-mm thoracic trocar was inserted in place of the $10-\mathrm{mm}$ umbilical trocar. The ELViS was then inserted and deployed under visual control, using the endoscope. The screens were placed to the left or to the right of the pig, depending on the experimental step being performed.

\section{Experimental protocol}

\section{Risk analysis}

Risk analysis represents an essential milestone in the preparation of a request to the competent authorities for authorisation to use a non-CE-labelled medical device in human clinical trials. Therefore, two preparatory sessions were performed to validate the safety of use of the ELViS, and to ensure that the material was functional (initial validation of the proof of concept) and that the experimental protocol (chosen exercises) conformed to general surgical practice. A risk analysis plan was defined with the assistance of a company specialising in regulatory affairs (SQI, SurgiQual Institute, Grenoble, France), acting as a partner in the project. The criteria studied were: safety during insertion, deployment/folding of the camera module, and use of the ELViS, with the camera module in contact with the viscera (extremities of the ELViS) and in all orientations in the abdominal cavity. Each step was scheduled to be repeated 20 times. An extraction procedure (small midline laparotomy performed under visual observation) was included in case of rupture of the device and performed during each preparatory session.

\section{Proof of concept}

Each experimental session was designed to involve a two-person team. Both participants in each team were expert laparoscopic surgeons. Three exercises (Figure 3) were proposed to reflect the required technical skills for general laparoscopic surgery. Each participant acted either as the lead surgeon or the assistant surgeon (laparoscope holder). One investigating surgeon (BT) monitored the session's 
progression and ensured that the ELViS was correctly installed. An additional investigating surgeon (AM) and an ergonomist (CS) were responsible for data collection. A computer science engineer (SV, MT) monitored the video feed to ensure it was functioning correctly.

After presenting the ELViS and the experimental model, the exercises were explained to participants on an anatomical representation of a dissected pig illustrating the anatomical specificities compared to human anatomy. During a period of familiarisation, the participants were asked to dissect the bladder or perform a cholecystectomy with the system and were encouraged to use the side screens to better understand the visual interface. The familiarisation lasted at least $15 \mathrm{~min}$ for each participant, or until they felt comfortable with the User Interface. A demonstration of each exercise is available in the supplementary video.

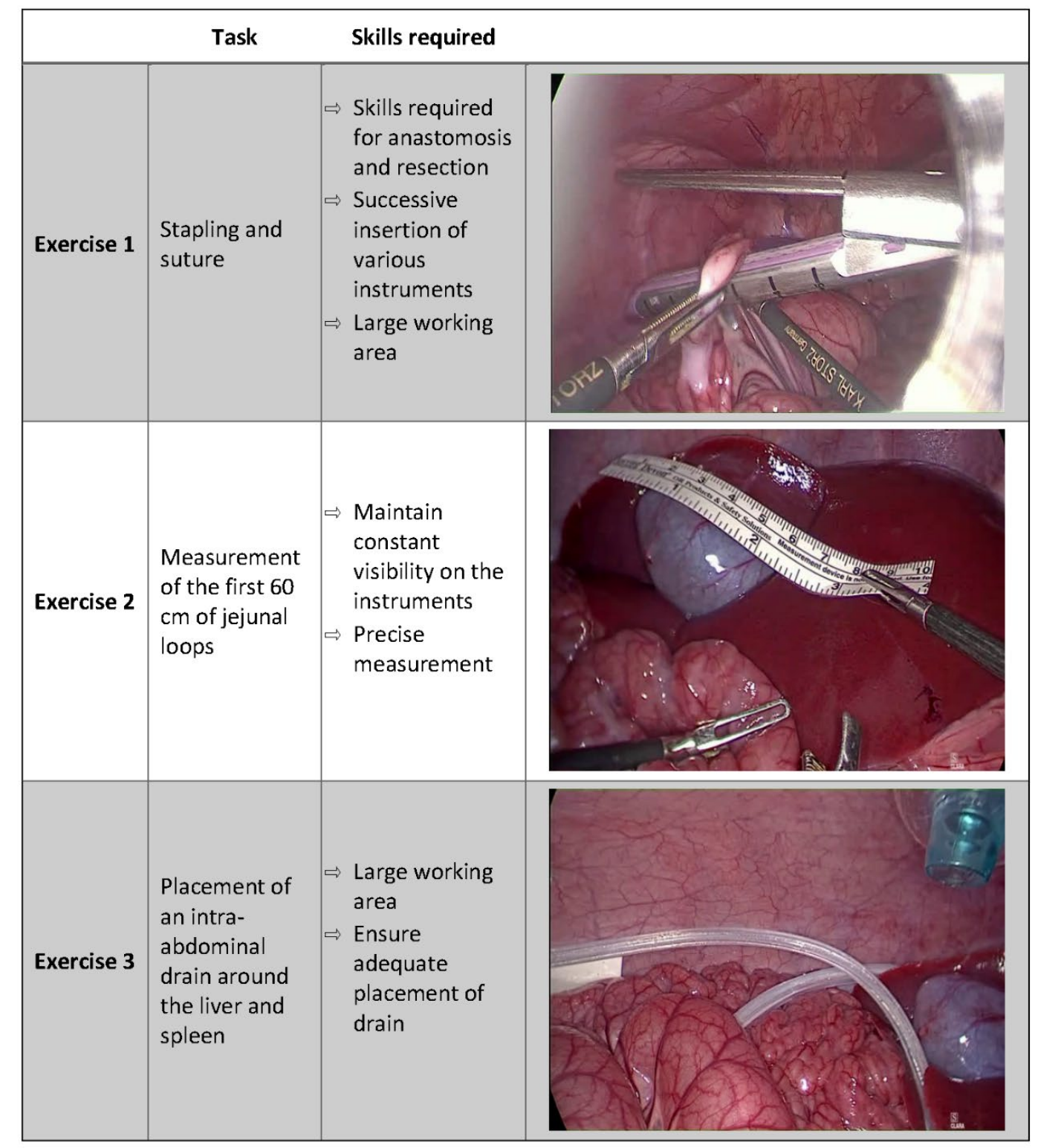

Figure 3. Description of the exercises and related skills necessary. 
Each participant performed the series of exercises, assisted by their designated partner, with the ELViS providing complementary images in addition to those provided by the endoscope, or with the endoscope alone. The order in which exercises were performed was chosen so as to limit the learning bias for the exercise: participants did not systematically use the same method the first time they performed an exercise. For example, for the first exercise, during the first round, the first participant would perform as operating surgeon with the ELViS and the second participant without. Then for the second round of the first exercise, the first participant would perform as operating surgeon without the ELViS and the second participant would operate with the ELViS. The order was switched for the two rounds of the second exercise. The participant who started the session was selected randomly. At the end of the session, each participant had performed each exercise with and without the ELViS.

\section{a) Exercise 1}

The first exercise aimed to assess the suture, stapling and knot tying skills required to perform an intestinal resection and anastomosis (gastric bypass type). The operator was positioned to the right of the pig and the tower to the left. A loop of the distal small intestine which could be sutured to the bladder had to be identified. After opening the meso with monopolar scissors, the small intestine was sectioned with the help of a laparoscopic stapler. The digestive segment was attached to the vesical peritoneum using an intra corporeal stitch with resorbable braided filament $3 / 0$. Bowel anastomosis was not performed so as to be able to use the same animal for several rounds without soiling of the surgical field. The exercise was finished when three knots had been firmly tightened.

During this exercise, several instruments were successively inserted via the trocars, which were not visible in the conventional endoscopic image (risk of intestinal perforation at each insertion). The work area was quite large as the small intestine had to be mobilised and then moved down in the pelvis, and peripheral vision could be useful during knot tightening when placing the stitch.

\section{b) Exercise 2}

During the second exercise, the operator used an endoscopic ruler to measure $60 \mathrm{~cm}$ of intestine from the first mobile jejunal loop. The operator was positioned to the left of the pig and the tower to the right. The exercise was completed after the last bowel segment had been displaced near the intraabdominal ruler. This manoeuvre is commonly performed in laparoscopic surgery during gastric bypass interventions. It requires the intestines to be unrolled (during this stage, surgical instruments are frequently lost to view); the intestine to be measured must be constantly visible, as must the ruler. Intestinal injuries are a known risk during grasping and mobilisation of portions of the digestive tract[20]. 


\section{c) Exercise 3}

During the third exercise, the surgeon placed a $60-\mathrm{cm}$ flexible silicon drain in a difficult-to-access zone (around the liver and spleen). This exercise required the operator to maintain overall visibility of the drain to be sure that it remained in place during manipulation (e.g. behind the liver as it was placed under the spleen). The exercise was completed when the drain was correctly positioned between the lobes of the liver, under the spleen, and the two extremities were brought together under the liver. This procedure can be particularly complex in laparoscopic surgery in humans, as it takes place at the end of the intervention (risk of damaging previous intervention site). In addition, drain tubing is made of siliconized plastic that is both flexible and elastic. For this reason, the drain may move from the desired trajectory while the tip is placed in the desired area. A broader field of vision could help to better verify the position of the drain at all times.

\section{Debriefing session}

At the end of the experiment, an ergonomist conducted a structured interview with the participants [21]. The session was recorded using a Dictaphone and took place in two parts. Firstly, the System Usability Scale [22] (SUS) was proposed to establish a reference usability score. SUS is a reliable tool for measuring the usability of surgical devices. Participants are asked to score 10 items on a Likert scale. The survey is neutral as 5 items are positively oriented and 5 items negatively oriented. This questionnaire provides a score between 0 and 100, which can be compared to a threshold scale [23]. For instance, on nearly 1000 SUS surveys, Bangor et al. [23] examined the relationship between the SUS scores and the following adjective ratings: "Worst Imaginable", "Awful", "Poor"; "OK"; "Good"; "Excellent"; and "Best Imaginable". They concluded that a score above 71.4/100 corresponded to a "Good" usability of the device, whereas a score above 85.5/100 reflects "Excellent" usability of the device. The score determined here will serve as a reference for future versions of the ELViS system. Following recording of the SUS, a semi-structured interview was conducted. An interview guide was prepared in advance to direct the themes of the discussion, based on open questions (supplementary material). Closed questions were used in a subsequent phase to refine or validate specific elements. Qualitative responses given by surgeons were subsequently analysed from the recording of the exchanges during the interview.

\section{Assessment criteria}

The duration of the intervention was the main quantitative criterion used for this study. Time was measured after the introduction of the first instrument until the exercise was completed. The occurrence of any adverse events was noted. 
An external camera was used to record the operating theatre throughout the session. All verbal and non-verbal exchanges were analysed a posteriori by an ergonomist (CS).

During the debriefing, the SUS score was calculated.

\section{E. Statistical analysis and regulatory aspects.}

The experimental protocol was approved by the local ethics committee (No. APAFIS\#18285$2018122718408122 \mathrm{v} 1$ ) and conformed to the 3R principles (Replacement, Reduction and Refinement) described by Russel and Burch [24], aiming to reduce the number of animals necessary to answer a scientific question, when an animal model is essential, and to ensure the well-being of the animal during the experiment. Each participant signed a consent form (study, video and audio recording) and a confidentiality agreement.

Statistical analysis was performed using STATACORP 2017 (Stata Statistical Software: Release 15. College Station, TX: StataCorp LLC) software. Times were reported in seconds. A nonparametric Wilcoxon test was used to compare mean times-to-completion. A threshold of $5 \%$ (alpha risk) was used to determine the statistical significance of results ( $p$-value).

\section{Results}

\section{A. Risk analysis}

During the two preparatory sessions, the ELViS prototype allowed surgeons to perform surgical tasks without technical failures on the animal model. The cameras provided lateral images throughout the duration of the experiment without loss of data. The device was inserted, deployed under the control of the endoscope or blindly, and withdrawn without difficulty over the 20 repetitions for each preparatory session according to the risk analysis plan designed by SurgiQual Institute. Endoscope insertion was conducted as usual, with normal sliding and without excessive friction.

An acceptable pneumoperitoneal volume was maintained throughout the surgical intervention.

The safety test phases revealed no risk-associated events. The ELViS was mobilised along with the camera module throughout the abdominal cavity, including in contact with hollow organs. For instance, the device was deployed and folded in contact with the small intestine. No damage due to ripping or pinching was recorded. No risk-associated event was identified, in particular during withdrawal of the ELViS under visual control.

The withdrawal procedure in case of rupture of the material required extension of the median periumbilical incision. The still-deployed ELViS could then be withdrawn without difficulty. 


\section{B. Proof of usability and Assessment of the ELViS on pigs}

\section{Study participants}

Seven volunteers from two university teaching hospital centres (Grenoble-Alpes hospital centre and Lyon civic hospices) participated in the study, spread over four sessions (four pigs) which took place between April and June 2019 at the School of Surgery at the University of Lyon. The level of laparoscopy expertise was high (three second-year fellows in laparoscopic surgery, two hospital practitioners, and two university professors). Five of the surgeons were specialised in digestive surgery and two in urology. All participants habitually perform more than 100 laparoscopic interventions every year. All surgeons were familiar with the porcine animal model. Initially, eight surgeons were recruited for the experiment, but one cancelled their session the day before. The unpartnered surgeon therefore performed each exercise twice to comply with the experiment plan, and was assisted by one of the investigating surgeons (AM). Therefore, in total, eight equivalent-participants were assessed.

\section{Exercises}

All exercises were completed by the participants. Mean time-to-completion did not differ significantly between the ELViS and conventional endoscopy conditions for any of the exercises. Table 2 presents the mean times-to-completion for each exercise.

\begin{tabular}{|l|l|l|l|}
\hline & \multicolumn{2}{|l|}{ Mean time (s, IQR 25-75) } & \\
\hline Exercise & ELViS & Endoscope only & $\mathrm{p}$ \\
\hline & & & \\
\hline Total & $187.5(50-250)$ & $158.5(94.5-262)$ & 0.833 \\
\hline $\mathbf{1}$ & & & \\
\hline & $289.5(206-347)$ & $284(233-325)$ & 0.780 \\
\hline $\mathbf{2}$ & & & \\
\hline $\mathbf{3}$ & $61.5(53-96)$ & $77(64.5-101)$ & 0.674 \\
\hline & & & 0.886 \\
\hline
\end{tabular}

Table 2. Mean time required to complete exercises.

One intestinal injury (small intestine perforation due to grasping and tearing with laparoscopic forceps) was recorded during grasping of the intestine as part of Exercise 2 without the ELViS. No other undesirable event was identified during the sessions. No complications regarding the use of ELViS were identified during the experiments. 


\section{SUS results and interviews}

After finishing the exercises, all participants completed the SUS. The mean SUS score determined from the responses to questionnaires was 74.7. The SUS score is detailed by item in Figure 4.
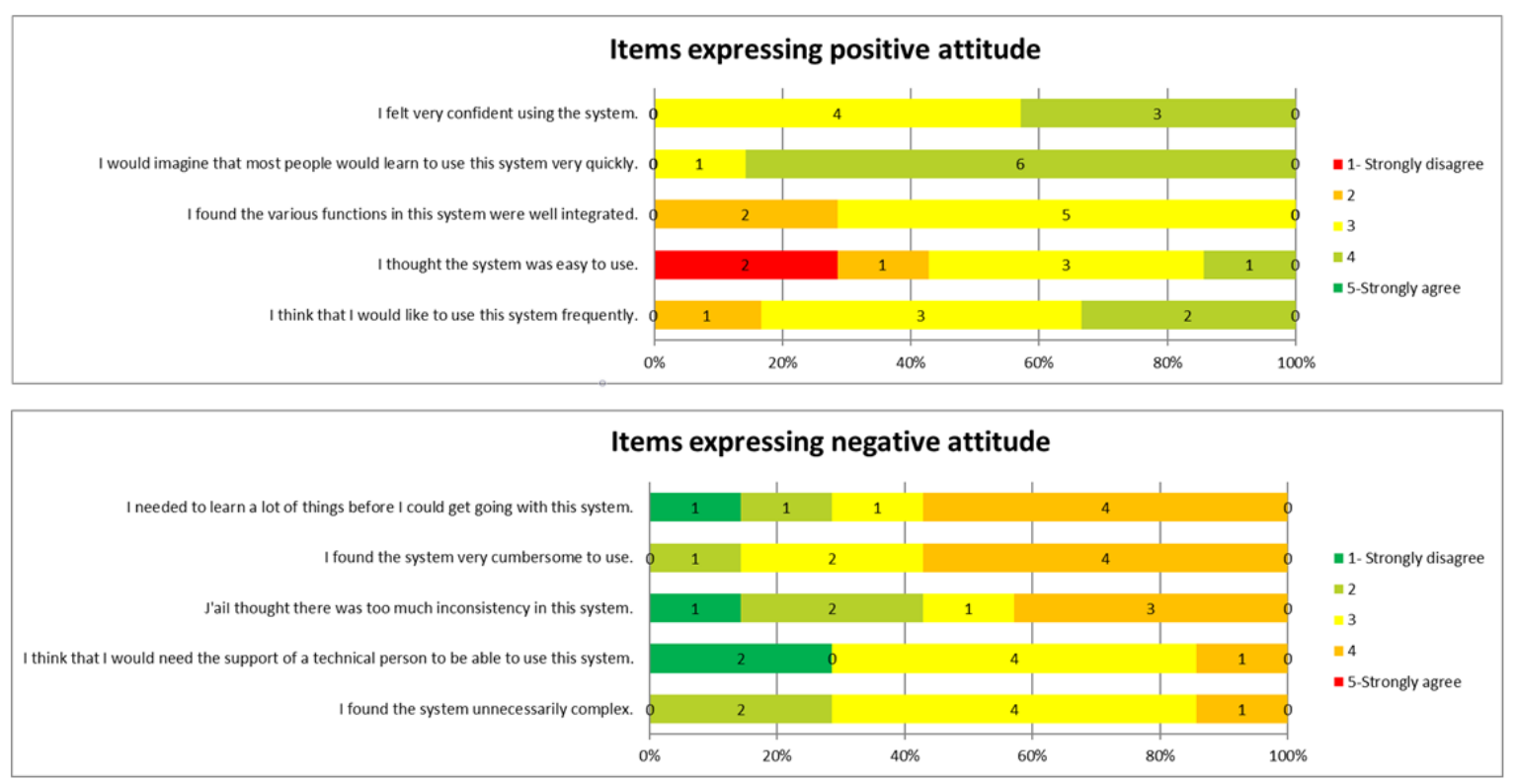

Figure 4. Responses of the participants to each item. The colour represents the answer on the Likert scale. The number of participants providing each answer is indicated for each item. Items expressing a positive or negative attitude are represented together but were recorded alternatively.

The seven participants' feedback was then obtained through semi-structured interviews. These interviews provided information on the four topics addressed: installation, use, withdrawal of the system, and whether surgeons projected to use the system with real patients.

The discussions on the installation of the system provided references of other medical devices that could be used as examples for improving the ELViS, such as cholangiography kits with a convenient button (like the Aerostat ${ }^{\circledast}$, Applied Medical, California, USA), or single-use ureteroscopes to avoid sterilisation issues.

With regard to the usability of the system, surgeons highlighted the added information provided by the lateral cameras without additional work load. To do so, they used phraseology such as "I looked at it a lot", "We do not need to look at it [the side camera image] all the time but only take a glance to check if the drain is well placed". Having the three images merged on an single screen, or the same image quality (High Definition) on the three screens were considered as important visual improvements. The withdrawal of the system raised no concerns for any of the participants.

Participants stated the surgical procedures for which the ELViS would be most helpful. The most frequently reported were laparoscopic or robot-assisted rectal resection, partial nephrectomy, pyeloplasty, bypass surgery, and complex liver surgeries. 
The participants pointed out the benefit that this system could provide for the safety of procedures thanks to the additional views. One surgeon recounted the experience of a trocar inserted too laterally that caused an iliac vein injury that could have been prevented if this kind of device had been used. The situations identified by the surgeons where the ELViS could present a real advantage were the following:

- To explore the abdomen

- During instrument insertion

- When placing stitches, as the operator constantly has visibility of the needle and free thread

- For highly magnified surgery, as an aid to orientation at any time (mainly for the assistant)

- To allow constant visual control for the assistant responsible for placing the endoscope and additional laparoscopic forceps.

\section{Discussion}

For this article, an innovative trocar providing improved vision for the surgeon and surgical assistant was developed and used safely on one of the most reliable experimental models for laparoscopy, namely the porcine model.

The preclinical study phase allowed us to develop the reusable ELViS prototype, and to confirm the concept of use of the ELViS in the cadaveric human model and live pig model. We thus assessed potential risks for patients and identified areas where improvements would be of interest. Maturation of the ELViS is in phase 0 as described by the IDEAL framework recommendations $[25,26]$, as the idea for the innovation has been generated and a prototype developed. Several challenges remain to be addressed before tests in humans will be possible, in particular electronic compatibility and sterilisation.

Several teams have previously tackled the problem of restricted visibility in laparoscopy (Table 3).

\begin{tabular}{|l|l|l|}
\hline Innovations & Description & Weak points \\
\hline Suzuki et al. [25] & $\begin{array}{l}\text { Mini cameras deployed over a 5-cm } \\
\text { radius }\end{array}$ & $\begin{array}{l}\text { Absence of conventional } \\
\text { endoscope for high definition } \\
\text { image } \\
\text { Real-time vision difficult }\end{array}$ \\
\hline Silvestri et al. [26] & $\begin{array}{l}\text { Array of mini cameras introduced into } \\
\text { the abdominal cavity and } \\
\text { magnetically maintained }\end{array}$ & $\begin{array}{l}\text { System alignment } \\
\text { Introduction of the system } \\
\text { Absence of conventional } \\
\text { endoscope for high definition } \\
\text { image }\end{array}$ \\
\hline
\end{tabular}




\begin{tabular}{|l|l|l|}
\hline MARVEL [27, 28] & $\begin{array}{l}\text { Robotic camera bound to the } \\
\text { abdominal wall moved using an } \\
\text { external joystick }\end{array}$ & $\begin{array}{l}\text { Lack of fluidity during use } \\
\text { Image quality } \\
\text { Absence of conventional } \\
\text { endoscope }\end{array}$ \\
\hline $\begin{array}{l}\text { Naya et al. [29] } \\
\text { panoramic image }\end{array}$ & $\begin{array}{l}\text { On-demand panoramic } \\
\text { reconstruction by sweeping the } \\
\text { endoscope }\end{array}$ & Lack of real-time vision \\
\hline $\begin{array}{l}\text { Trocar-Camera } \\
\text { Assembly [30] }\end{array}$ & $\begin{array}{l}\text { Deployment of four mini cameras } \\
\text { around a laparoscopy trocar }\end{array}$ & $\begin{array}{l}\text { Fragility of the system for } \\
\text { clinical use } \\
\text { Image overlap conflict }\end{array}$ \\
\hline $\begin{array}{l}\text { Bird View System [31] } \\
\text { Global Vision } \\
\text { System[32] }\end{array}$ & $\begin{array}{l}\text { Moulded block with one or two wide- } \\
\text { angle camera placed at a distance } \\
\text { from the surgical area allowing wide- } \\
\text { angle vision. }\end{array}$ & $\begin{array}{l}\text { Lack of precision in selecting } \\
\text { the surgical area } \\
\text { Risk of occlusion of the image } \\
\text { by the endoscope and other } \\
\text { instrumentsPotential } \\
\text { confounding point of view }\end{array}$ \\
\hline
\end{tabular}

Table 3. Main medical devices developed to improve vision during laparoscopy.

Suzuki et al. [27] and Silvestri et al. [28] described systems introducing several miniature cameras into the abdomen in order to obtain a wider view without the use of the conventional endoscope. The image from each camera is displayed in a separate window. These devices provide numerous images displayed in different windows, which may increase mental workload in clinical practice, and possibly make the intervention more difficult as the surgeon has to integrate multiple sources of information. In addition, treatment of several images, for example for reconstructions, can require considerable calculation time, making real-time use impossible. Finally, the resolution of the miniature cameras used may be insufficient to perform surgery on a patient due to the lack of feed from a high-definition endoscope. To overcome this limitation, we developed ELViS as a complement to a conventional endoscope.

Castro et al. [29] described the MARVEL (Miniature Anchored Robotic Videoscope for Expedited Laparoscopy) system. In this device, an automated wireless camera is attached to the abdominal wall and controlled by the surgeon using a joystick. During animal experiments [30], the number of conflicts between instruments and the workload was lower in the group using the device due to the enhanced view. However, the need to manually change the camera's orientation to focus on the location where the dissection was to be performed could slow down the workflow and impede use of the system in clinical practice.

A panoramic reconstruction of consecutive laparoscopy images can be created using commercially available panoramic imaging software. Naya et al. [31] described the use of panoramic images in urological surgery. At any time during the intervention, the surgeon can acquire a panoramic image of the surgical area by sweeping the endoscope from one side of the area to the other. The image is 
displayed on a reminder screen and can be used to help guide the surgical procedure. During a clinical study on nephrectomy with and without panoramic vision [31], the time-to-completion and average bleeding favoured interventions where panoramic vision was available. However, this method cannot provide a real-time image of the abdominal cavity as the surgeon has to update the panoramic view, by sweeping the endoscope once again, at every significant change of the surgical field.

Since the start of the project to develop the ELViS, several other distributed vision systems have been described. Kim et al. [32] described the Trocar-Camera Assembly, which is similar to the ELViS as it includes a conventional endoscope. This system uses four small cameras deployed around an optical laparoscopy trocar on thin aluminium supports. Test bench tests allowed simple tasks to be performed (object transfer) with a broader vision of the surgical area thanks to a representation showing a merge of the five images. To our knowledge, the solidity of the device and the robustness of the algorithm merging the images were not tested on cadavers or living organisms. In a similar vein, the prototype presented by Tamazdate et al. [17] was too fragile to be useful in a clinical setting.

Other distributed vision systems, such as that developed by Rivas-Blanco et al. [34] and the Bird View System developed by Sumi et al. [33], provide a point of view that differs significantly from that provided by the conventional endoscope. These devices are inserted into the abdominal cavity and placed in contact with the abdominal wall away from the area where the intervention takes place. The wired connections to the screen exit through a small trocar. The surgeon can perform surgery with the conventional endoscope and the help of the image from the device, which provides a broader vision of the abdominal cavity. These approaches were clinically validated during urological or colorectal procedures. They have the advantage of providing a point of view from behind the inserted endoscopic camera, and thus help to eliminate all blind spots. However, some concerns may be raised about their use in real-life surgery. First, the devices are positioned far from the surgical field, and the size of the instruments visible on the side screen might be too small to provide relevant information. Moreover, the authors did not discuss how the orientation of the camera could be changed, or if they needed to be repositioned using the laparoscopic forceps to ensure that the image would always be centred on the operating field. Indeed, during laparoscopic surgery, the location of the endoscope usually changes several times, and it is important that any additional camera and the endoscope have similar orientation. The cognitive impact of presenting images from very different perspectives was not discussed in these previous studies.

The ELViS presented here addresses the issue of the field of vision in laparoscopy without obviating the need for an endoscope, unlike the first devices presented above [27-29, 31]. The endoscope and the additional cameras have similar fields of vision and orientation (aligned with the trocar), unlike the 
Birdview [33] and Rivas-Blanco's Global Vision System [34]. The point of view for the side cameras in the ELViS is at a distance from the surgical area and therefore allows a broader vision. The option to have the same orientation between the distributed laparoscopy cameras and the endoscope was chosen as the display seemed more natural and we considered it would limit the mental workload required to integrate the visual information provided by the different images during surgical interventions. Indeed, one of the basic concepts of laparoscopy is triangulation. To have the fewest constraints possible during handling of the instruments and the best $3 \mathrm{D}$ reconstruction, the working trocars must be placed to either side of the optical trocar. In distributed laparoscopy, cameras tethered to the abdominal wall provide an unusual point of view, and, significantly, all the cameras have different angles. The presentation of the images acquired therefore becomes complex. Lastly, we chose to design the ELViS as a modified trocar so that the horizon lines for all the images could be effectively matched by twisting the device slightly to align it with the endoscope image. Any misplacement can thus be rapidly corrected.

During this proof of concept, the use of the ELViS did not negatively impact the time-to-completion for the procedures tested during the exercises. The lack of difference in terms of time-to-completion is in line with the results of experiments on test benches performed previously (unpublished results). The Time-to-completion criterion was selected here as it was a simple means to assess the proof of concept for the use of a medical device (MD). It will not be possible to isolate this parameter during future phase 1 testing in humans, and it will therefore be associated with criteria relating to the quality of the surgery, such as the surgical team's satisfaction or peri-operative complications, which are difficult to assess in the experimental model used here. The best quantitative parameter by which to assess the ELViS is difficult to determine. Indeed, the time-to-completion is an imperfect criterion as its meaning in laparoscopic surgical practice is medico-economic. The clinical advantage of an improvement in time of a few minutes for an intervention lasting several hours is probably negligible. In contrast, the time to react to an adverse event could be of interest. Nevertheless, serious adverse events are rare in laparoscopy, and this criterion could therefore not be used in isolation. Finally, scores that assess surgical performance (GOALS [35], OSATS [36], etc.) were not ideally suited to our experiment as the times-to-completion were short, and the number of iterations small. In this series of experiments, the relatively low number of participants prevented us from fully assessing surgical performance. The number of movements of the endoscope or the assistant's quality, as determined by a validated score (e.g. SALAS [37]), would allow us to assess the impact of the ELViS on the person holding the camera. Qualitative assessments (user debriefing) by an ergonomist were more informative and appeared better adapted to assess this device at this proof-of-concept stage. 
The usability of the ELViS was studied based on the SUS scores and the debriefing sessions with the experts. The SUS mean score (74.7) allows us to class the usability of the ELViS as "good" [23]. The limitation with this score is the small number of responses (eight equivalent-participants). Analysis of the SUS score by item (Figure 4) highlighted a few points raising questions, which included simplicity of use, the learning curve for the system and the constraints imposed in the context of the operating theatre (placement of supplementary screens).

The debriefing sessions with the experienced laparoscopic surgeons indicated a positive attitude towards the system. The lexicon used by surgeons included statements such as "I am thrilled to see my stapler as it is introduced". During these sessions, points for improvement were also identified. Firstly, the setup was considered to be too cumbersome. Therefore, we plan to work on miniaturization and improving the quality of the implanted camera. The reduced size could make it possible to add an integrated system to modify the angle of the side cameras. In addition, we wish to implement wireless video-feed transmission to eliminate the wire from the abdomen. The User Interface will be improved by including the video-feed from the side cameras and the endoscope on a single wide screen.

One of the limitations of our assessment was the use of the pig as a model. Indeed, after insufflation, the distance between the abdominal wall and the surgical area is shorter in pigs than in humans. However, the ELViS presents its greatest advantage when the distance between the extremity of the endoscope (zoomed in on the surgical area) and the lateral cameras (placed against the abdominal wall) is as large as possible to take advantage of a maximum of perspective. Thus, the effect of an overall view could be more dramatic in laparoscopy in live humans than in the pig.

The ELViS could improve the workload in laparoscopy. All the constraints inherent to minimally invasive surgery significantly increase the mental workload and fatigue for the surgeon, whatever their level of experience [38, 39]. On a population of 28 surgeons performing a series of knots on a laparoscopy or open surgery simulator [38], the workload, the level of concentration, and stress were all higher with laparoscopy. This effect was less pronounced in an expert population. Law et al. [39] assessed workload using the NASA Task Load [40] during 238 colorectal surgeries performed as open surgery, laparoscopy or robot-assisted surgery. The overall, mental, and physical workloads and the level of concentration required were all significantly higher for laparoscopy than for open surgery or robot-assisted surgery. These aspects will be assessed with the ELViS in future clinical studies.

The ELViS could also be used for other minimally invasive procedures. Teleguided robot-assisted surgery was popularised by Lindbergh's transatlantic surgical intervention [41]. Teleguided surgery has mainly developed over the last decade with the emergence on the market of the Da Vinci Robot (Intuitive Surgical ${ }^{\circledR}$, Sunnyvale, United States), which consists of three or four sterile articulated-arms and a command module with a non-sterile User Interface through which the surgeon performs their 
intervention. To perform some movements, the surgeon must zoom in on the surgical area where they are operating, thus losing visibility of the teleguided arms and the instruments manipulated by their assistant. Vascular [42], urethral [43] or intestinal [44] injuries in robot-assisted abdominal surgery can occur during instrument insertion, as a result of a dysfunctional instrument (electric arc), poor demonstration by the assistant, or inappropriate dissection. As the surgeon is not next to the patient, they cannot respond rapidly to (extremely urgent) situations, for example by rapidly redirecting the camera to view a particular area and intervene using instruments that are already to hand. Mobilisation of the robot-assisted camera is less instinctive, and although instrument mobilisation is more precise, it is considerably slower than with manual control. This situation creates additional anxiety as the surgeon feels that they are not fully in control of the intervention. The advantages of the ELViS could thus be even more pronounced in robot-assisted surgery.

\section{Conclusion}

In conclusion, the ELViS developed in the TIMC laboratory was successfully used on a live laparoscopic model. Feedback from expert surgeons was promising. The next stage in development will be to demonstrate feasibility in humans after adaptation of the device through electronic and sterilization compatibility.

\section{Acknowledgements}

The authors would like to thank Prof. Jean Luc Bosson and Mr Aboubacar SISSOKO (data stat cell, CHU Grenoble) for their expertise and contribution to the statistical analysis of the data, and Maighread Gallagher-Gambarelli (TWS Scientific Editing) for suggestions on English usage in the paper. The authors would also like to thank the French National Research Agency for funding this work through the DEPORRA2 project - reference ANR-14-CE17-0009, the CQFD LABCOM project - reference ANR-13-LAB3-0002, and the Investissements d'Avenir programme (Labex CAMI - reference ANR-11LABX-0004).

\section{Disclosures}

Dr. Sandrine Voros has registered a patent EP2903495B1 (FR2996437B1). The work described in this paper was funded by the French National Agency for Research (Agence Nationale de la Recherche) as part of the DEPORRA2 project (reference ANR-14-CE17-0009), by the the CQFD LABCOM project (reference ANR-13-LAB3-0002), and by the French government's Investissements d'Avenir programme as part of the Labex CAMI (reference ANR-11-LABX-0004). 
Sandrine VOROS, Bertrand TRILLING, Adrian MANCINI, Gaëlle FIARD, Pierre Alain BARRAUD, Marion DECROUEZ, Sinara VIJAYAN, Mathias TUMMERS, Jean Luc FAUCHERON, Sophie SILVENT, Christel SCHWARTZ have no other conflicts of interest or financial ties to disclose. 


\section{Bibliography}

1. Tanis PJ, Buskens CJ, Bemelman WA (2014) Laparoscopy for colorectal cancer. Best Pract Res Clin Gastroenterol 28:29-39 . https://doi.org/10.1016/j.bpg.2013.11.017

2. van der Pas MH, Haglind E, Cuesta MA, Fürst A, Lacy AM, Hop WC, Bonjer HJ (2013) Laparoscopic versus open surgery for rectal cancer (COLOR II): short-term outcomes of a randomised, phase 3 trial. The lancet oncology 14:210-218

3. Anderson SA, Beierle EA, Chen MK (2014) Role of laparoscopy in the prevention and in the treatment of adhesions. Semin Pediatr Surg 23:353-356. https://doi.org/10.1053/j.sempedsurg.2014.06.007

4. Bonjer HJ, Deijen CL, Abis GA, Cuesta MA, van der Pas MHGM, de Lange-de Klerk ESM, Lacy AM, Bemelman WA, Andersson J, Angenete E, Rosenberg J, Fuerst A, Haglind E (2015) A Randomized Trial of Laparoscopic versus Open Surgery for Rectal Cancer. New England Journal of Medicine 372:1324-1332 . https://doi.org/10.1056/NEJMoa1414882

5. Ratti F, Fiorentini G, Cipriani F, Catena M, Paganelli M, Aldrighetti L (2018) Laparoscopic vs Open Surgery for Colorectal Liver Metastases. JAMA Surgery 153:1028 . https://doi.org/10.1001/jamasurg.2018.2107

6. McMahon AJ, Fullarton G, Baxter JN, O'Dwyer PJ (1995) Bile duct injury and bile leakage in laparoscopic cholecystectomy. British Journal of Surgery 82:307-313 . https://doi.org/10.1002/bjs.1800820308

7. Pesce A, Palmucci S, La Greca G, Puleo S (2019) latrogenic bile duct injury: impact and management challenges. Clinical and Experimental Gastroenterology Volume 12:121-128 . https://doi.org/10.2147/CEG.S169492

8. Sammour T, Kahokehr A, Srinivasa S, Bissett IP, Hill AG (2011) Laparoscopic Colorectal Surgery Is Associated With a Higher Intraoperative Complication Rate Than Open Surgery: Annals of Surgery 253:35-43 . https://doi.org/10.1097/SLA.0b013e318204a8b4

9. Cardin J, Johanet H (2016) For a Better Evaluation of Intraoperative Adverse Events in Laparoscopic Surgery: The Club Cœlio Experience. Advanced Laparoscopy

10. on behalf of the EAES committees, Francis NK, Curtis NJ, Conti JA, Foster JD, Bonjer HJ, Hanna GB (2018) EAES classification of intraoperative adverse events in laparoscopic surgery. Surgical Endoscopy 32:3822-3829 . https://doi.org/10.1007/s00464-018-6108-1

11. Curtis NJ, Dennison G, Brown CSB, Hewett PJ, Hanna GB, Stevenson ARL, Francis NK (2019) Clinical Evaluation of Intraoperative Near Misses in Laparoscopic Rectal Cancer Surgery: Annals of Surgery 1 . https://doi.org/10.1097/SLA.0000000000003452

12. Cahais J, Schwarz L, Bridoux V, Huet E, Tuech J-J (2017) Is the image "right" for everyone? Introduction to the parallax effect in laparoscopic surgery. Journal of Visceral Surgery 154:1114. https://doi.org/10.1016/j.jviscsurg.2016.06.007

13. Wentink M, Breedveld P, Meijer DW, Stassen HG (2000) Endoscopic camera rotation: a conceptual solution to improve hand-eye coordination in minimally-invasive surgery. Minimally 
Invasive Therapy \& Allied Technologies 9:125-131.

https://doi.org/10.3109/13645700009063059

14. Conrad J, Shah AH, Divino CM, Schluender S, Gurland B, Shlasko E, Szold A (2006) The role of mental rotation and memory scanning on the performance of laparoscopic skills: A study on the effect of camera rotational angle. Surgical Endoscopy 20:504-510 .

https://doi.org/10.1007/s00464-005-0363-7

15. Trilling B, Vijayan S, Goupil C, Kedisseh E, Letouzey A, Barraud PA, Faucheron JL, Fiard G, Voros $S$ (2020) Enhanced Laparoscopic Vision Improves Detection of Intraoperative Adverse Events During Laparoscopy. IRBM S1959031820301846 . https://doi.org/10.1016/j.irbm.2020.12.001

16. Tamadazte B, Agustinos A, Cinquin P, Fiard G, Voros S (2015) Multi-view vision system for laparoscopy surgery. International Journal of Computer Assisted Radiology and Surgery 10:195203 . https://doi.org/10.1007/s11548-014-1064-2

17. Tamadazte B, Fiard G, Long J-A, Cinquin P, Voros S (2013) Enhanced vision system for laparoscopic surgery. Conf Proc IEEE Eng Med Biol Soc 2013:5702-5705. https://doi.org/10.1109/EMBC.2013.6610845

18. Voros S, Moreau-Gaudry A, Tamadazte B, Custillon G, Heus R, Montmasson M-P, Giroud F, Gaiffe O, Pieralli C, Fiard G, Long J-A, Descotes J-L, Vidal C, Nguyen-Dinh A, Cinquin P (2013) Devices and systems targeted towards augmented robotic radical prostatectomy. IRBM 34:139-146 . https://doi.org/10.1016/j.irbm.2013.01.014

19. Kilkenny C, Browne WJ, Cuthill IC, Emerson M, Altman DG (2010) Improving bioscience research reporting: the ARRIVE guidelines for reporting animal research. PLoS biology 8:e1000412

20. Barrie J, Russell L, Hood AJ, Jayne DG, Neville A, Culmer PR (2018) An in vivo analysis of safe laparoscopic grasping thresholds for colorectal surgery. Surg Endosc 32:4244-4250 . https://doi.org/10.1007/s00464-018-6172-6

21. Lallemand C, Gronier G (2016) Méthodes de design UX: 30 méthodes fondamentales pour concevoir et évaluer les systèmes interactifs. Eyrolles, Paris

22. Brooke J (1996) SUS-A quick and dirty usability scale. Usability evaluation in industry 189:4-7

23. Bangor A (2009) Determining What Individual SUS Scores Mean: Adding an Adjective Rating Scale. Journal of Usability Studies 4:10

24. Russell WMS, Burch RL (1959) The principles of humane experimental technique. London, Methuen

25. Hirst A, Philippou Y, Blazeby J, Campbell B, Campbell M, Feinberg J, Rovers M, Blencowe N, Pennell C, Quinn T, Rogers W, Cook J, Kolias AG, Agha R, Dahm P, Sedrakyan A, McCulloch P (2019) No Surgical Innovation Without Evaluation: Evolution and Further Development of the IDEAL Framework and Recommendations. Annals of Surgery 269:211-220 . https://doi.org/10.1097/SLA.0000000000002794

26. McCulloch P, Altman DG, Campbell WB, Flum DR, Glasziou P, Marshall JC, Nicholl J (2009) No surgical innovation without evaluation: the IDEAL recommendations. The Lancet 374:11051112 . https://doi.org/10.1016/S0140-6736(09)61116-8 
27. Suzuki N, Hattori A (2013) Development of New Augmented Reality Function Using Intraperitoneal Multi-view Camera. In: Linte CA, Chen ECS, Berger M-O, Moore JT, Holmes DR (eds) Augmented Environments for Computer-Assisted Interventions. Springer Berlin Heidelberg, pp 67-76

28. Silvestri M, Ranzani T, Argiolas A, Vatteroni M, Menciassi A (2013) A multi-point of view 3D camera system for minimally invasive surgery. Sensors and Actuators A: Physical 202:204-210 . https://doi.org/10.1016/j.sna.2012.12.015

29. Castro CA, Alqassis A, Smith S, Ketterl T, Yu Sun, Ross S, Rosemurgy A, Savage PP, Gitlin RD (2013) A Wireless Robot for Networked Laparoscopy. IEEE Transactions on Biomedical Engineering 60:930-936 . https://doi.org/10.1109/TBME.2012.2232926

30. Han WK, Tan YK, Olweny EO, Yin G, Liu Z-W, Faddegon S, Scott DJ, Cadeddu JA (2013) Comparison Between Magnetic Anchoring and Guidance System Camera-Assisted Laparoendoscopic Single-Site Surgery Nephrectomy and Conventional Laparoendoscopic SingleSite Surgery Nephrectomy in a Porcine Model: Focus on Ergonomics and Workload Profiles. Journal of Endourology 27:490-496 . https://doi.org/10.1089/end.2012.0484

31. Naya Y, Nakamura K, Araki K, Kawamura K, Kamijima S, Imamoto T, Nihei N, Suzuki H, Ichikawa $\mathrm{T}$, Igarashi T (2009) Usefulness of panoramic views for novice surgeons doing retroperitoneal laparoscopic nephrectomy: Panoramic views for novice surgeons. International Journal of Urology 16:177-180 . https://doi.org/10.1111/j.1442-2042.2008.02215.x

32. Kim J-J, Watras A, Liu H, Zeng Z, Greenberg J, Heise C, Hu Y, Jiang H (2018) Large-Field-of-View Visualization Utilizing Multiple Miniaturized Cameras for Laparoscopic Surgery. Micromachines 9:431 . https://doi.org/10.3390/mi9090431

33. Sumi Y, Egi H, Hattori M, Suzuki T, Tokunaga M, Adachi T, Sawada H, Mukai S, Kurita Y, Ohdan H (2019) A prospective study of the safety and usefulness of a new miniature wide-angle camera: the "BirdView camera system." Surgical Endoscopy 33:199-205 . https://doi.org/10.1007/s00464-018-6293-y

34. Rivas-Blanco I, Sánchez-de-Badajoz E, García-Morales I, Lage-Sánchez JM, Sánchez-Gallegos P, Pérez-del-Pulgar CJ, Muñoz VF (2017) Global vision system in laparoscopy. Actas Urológicas Españolas (English Edition) 41:274-278 . https://doi.org/10.1016/j.acuroe.2017.03.007

35. Vassiliou MC, Feldman LS, Andrew CG, Bergman S, Leffondré K, Stanbridge D, Fried GM (2005) A global assessment tool for evaluation of intraoperative laparoscopic skills. The American Journal of Surgery 190:107-113 . https://doi.org/10.1016/j.amjsurg.2005.04.004

36. Martin JA, Regehr G, Reznick R, MacRae H, Murnaghan J, Hutchison C, Brown M (1997) Objective structured assessment of technical skill (OSATS) for surgical residents. Br J Surg $84: 273-278$

37. Huber T, Paschold M, Schneble F, Poplawski A, Huettl F, Watzka F, Lang H, Kneist W (2018) Structured assessment of laparoscopic camera navigation skills: the SALAS score. Surgical Endoscopy 32:4980-4984 . https://doi.org/10.1007/s00464-018-6260-7

38. Berguer R, Smith WD, Chung YH (2001) Performing laparoscopic surgery is significantly more stressful for the surgeon than open surgery. Surgical Endoscopy 15:1204-1207 . https://doi.org/10.1007/s004640080030 
39. Law KE, Lowndes BR, Kelley SR, Blocker RC, Larson DW, Hallbeck MS, Nelson H (2018) NASATask Load Index Differentiates Surgical Approach: Opportunities for Improvement in Colon and Rectal Surgery. Annals of Surgery 271:906-912 .

https://doi.org/10.1097/SLA.0000000000003173

40. Hart SG, Staveland LE (1988) Development of NASA-TLX (Task Load Index): Results of Empirical and Theoretical Research. In: Advances in Psychology. Elsevier, pp 139-183

41. Marescaux J, Leroy J, Gagner M, Rubino F, Mutter D, Vix M, Butner SE, Smith MK (2001) Transatlantic robot-assisted telesurgery. Nature 413:379-380 . https://doi.org/10.1038/35096636

42. Sotelo R, Nunez Bragayrac LA, Machuca V, Garza Cortes R, Azhar RA (2015) Avoiding and managing vascular injury during robotic-assisted radical prostatectomy. Therapeutic Advances in Urology 7:41-48 . https://doi.org/10.1177/1756287214553967

43. Lee Z, Kaplan J, Giusto L, Eun D (2016) Prevention of iatrogenic ureteral injuries during robotic gynecologic surgery: a review. American Journal of Obstetrics and Gynecology 214:566-571 . https://doi.org/10.1016/j.ajog.2015.10.150

44. Picerno T, Sloan NL, Escobar P, Ramirez PT (2017) Bowel injury in robotic gynecologic surgery: risk factors and management options. A systematic review. American Journal of Obstetrics and Gynecology 216:10-26 . https://doi.org/10.1016/j.ajog.2016.08.040 